\title{
El aprendizaje servicio como propuesta metodológica para una pedagogía crítica
}

\author{
Òscar Chiva Bartoll, Jesús Gil Gómez, Raquel Corbatón Martínez, Carlos \\ Capella Peris \\ Universitat Jaume I. España
}

\section{Resumen}

El presente artículo aborda la relación entre la pedagogía crítica y los pilares fundamentales de la metodología del aprendizaje servicio. Su principal objetivo es demostrar, de un modo concluyente, la idoneidad del aprendizaje servicio como metodología para alcanzar objetivos pedagógicos tales como la emancipación, la transformación y la justicia social. El trabajo parte de la clarificación de los pilares fundamentales de la pedagogía crítica, entendida como paradigma filosófico-educativo que emerge de la Teoría Crítica de la Escuela de Frankfurt. Posteriormente, el artículo da cuenta de los fundamentos pedagógicos del aprendizaje servicio, aportando argumentos que evidencian su capacidad para satisfacer las metas pedagógicas de la pedagogía crítica. Por último, se ejemplifica dicha correlación a partir de una breve revisión de investigaciones sobre los efectos del aprendizaje servicio, así como a partir de la descripción detallada de una investigación llevada a cabo en el contexto español, que pone de manifiesto las posibilidades reales del aprendizaje servicio como metodología al servicio de la pedagogía crítica.

\section{Palabras clave}

Aprendizaje servicio, pedagogía crítica, metodología, educación, ciudadanía.

Fecha de recepción: 8/VIII/2014

Fecha de aceptación: 25/I/2015 


\section{Service learning as a methodological approach for the critical pedagogy}

\section{Abstract}

This paper describes the relation between the critical pedagogy and the fundamentals of the service learning methodology. Its main objective is to demonstrate, conclusively, the suitability of service learning as a pedagogical methodology to achieve objectives such as empowerment, transformation and social justice. Firstly, the paper clarifies the fundamentals of the critical pedagogy, understood as a philosophical-educational paradigm emerging from the Critical Theory of the Frankfurt School. Subsequently, the article reports on the pedagogical foundations of service learning, providing arguments that demonstrate their ability to meet the educational goals of critical pedagogy. Finally, this correlation is exemplified from a brief review of research on the effects of service learning, as well as from the detailed description of a research conducted in the Spanish context, highlighting the real possibilities of this methodology to achieve the aims of the critical pedagogy

\section{Keywords}

Service learning, critical pedagogy, methodology, citizenship, education. 


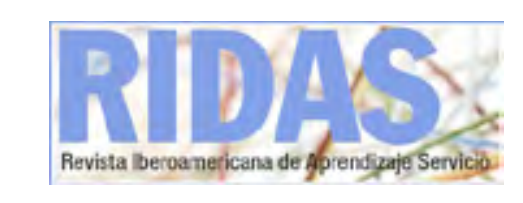

\section{Introducción}

La situación social actual, plagada de injusticias y desavenencias sociales, exige a gritos un cambio de paradigma educativo que potencie nuevas metodologías encaminadas a desarrollar al máximo la racionalidad crítica de las futuras generaciones. El momento que vivimos exige nuevas opciones para profundizar en los conocimientos personales y reforzar la identidad social del alumnado, permitiéndole así transformar la sociedad en la que vivimos y convertir el mundo en un lugar más humano.

Es necesario abogar por un paradigma pedagógico socio-crítico que parta de valores como el diálogo, la cooperación, la toma de decisiones, la reflexión, la comunicación democrática, etc. Un paradigma educativo que vele por la emancipación del individuo, a través del desarrollo del juicio moral y la crítica social; en el que el proceso sea tanto o más importante que el resultado, y que se centre en metas como el pleno desarrollo individual y colectivo de los educandos. En definitiva, un enfoque educativo que priorice la integración social, los valores cívicos, la relación justa entre iguales y la resolución de conflictos sociales.

No es novedad alguna la idea de transformar la sociedad a partir de mentes críticas y consecuentes, aunque esta concepción educativa no es la que generalmente vemos a nuestro alrededor ¿Por qué ocurre esto? ¿No hay metodologías adecuadas para ello? ¿Por qué siempre nos centramos en perpetuar la enseñanza de conocimientos técnicos y no en formar personas críticas? Es palmario que sí existen metodologías que abarcan cada uno de estos aspectos y que deberían ser consideradas como metodologías ligadas directamente a la pedagogía crítica (PC). Por nuestra parte, entendemos que una de las opciones metodológicas más apropiada para cumplir con las metas de este posicionamiento es el aprendizaje servicio (ApS). 
Con este artículo pretendemos clarificar la relación entre la PC y la metodología del ApS, mostrando así la idoneidad de esta nueva metodología para poner en práctica los atributos más esenciales de la PC. En primer lugar especificaremos los pilares fundamentales de la PC como paradigma filosófico-educativo que emerge con la Teoría Crítica. Posteriormente, tras revisar los fundamentos pedagógicos del ApS, argumentaremos su capacidad de satisfacer las metas pedagógicas socio-criticas. Finalmente, se ejemplificará dicha correspondencia a partir de la descripción de casos y resultados de aplicaciones concretas de ApS, poniendo de manifiesto sus posibilidades reales como metodología plenamente alineada con la PC.

\section{Pedagogía crítica}

Por teoría crítica se conoce la herencia del trabajo que los intelectuales de la escuela de Frankfurt desarrollaron a principios y mediados de siglo XX. Su legado se ha centrado en una crítica, al unísono, de elementos como la racionalidad instrumental, la industria de la cultura, el autoritarismo, la separación entre la teoría y la práctica, etc.

Habitualmente, a pesar de mantener profundas divergencias con sus predecesores, se considera al filósofo alemán Jürgen Habermas como el último miembro de la escuela de Frankfurt. Este autor, sin embargo, es uno de los que más influencia ha tenido sobre la corriente pedagógica crítica. En resumidas cuentas, Habermas (1982, 1989, 1991, 2002) considera que la racionalidad contemporánea es discursiva y se basa en la intersubjetividad y la intercomunicación, siendo estos elementos indispensables para la convivencia y el progreso humano. De ahí que centre su trabajo en el concepto de acción comunicativa, entendida como comunicación desarrollada en contextos sociales en los que el objetivo es la comprensión mutua, en contraposición con la comunicación estratégica, basada en 
perseguir el éxito al margen de los intereses legítimos de los interlocutores.

Otro concepto básico para comprender mejor los planteamientos de este autor, como indica LópezPastor (2002), es el de los intereses constitutivos del saber. Habermas (1982) diferencia entre tres tipos de intereses que condicionan la elaboración del conocimiento y saber humanos: (1) el interés técnico, (2) el interés práctico y (3) el interés emancipatorio.

El primero de ellos es de carácter instrumental, y se centra en conocer y dominar determinados medios (instrumentos) para alcanzar ciertos fines. Por otra parte, el interés práctico, basado en la razón práctica, es de carácter ético y busca guiar, orientar e informar a las personas en los procesos morales de toma de decisión. Finalmente, el interés emancipatorio, persigue los valores de justicia, libertad e igualdad, buscando la superación de las relaciones de poder, dependencia, dominio y/o marginación existentes entre los seres humanos, en tanto que seres inevitablemente sociales.

A raíz de estos planteamientos, la $\mathrm{PC}$ rechaza el enfoque de la didáctica entendida como una tecnología, en la que la educación esté en manos de docentes con un carácter técnico, que no se cuestionen los fines, los valores ni el para qué de su acción pedagógica. La educación debe tener, según el planteamiento crítico, una identidad emancipatoria clara, basada en el desarrollo del entendimiento y de la autonomía como atributos necesarios para derrocar el sometimiento y la manipulación estratégica de unas personas respecto a otras. Esta manera de hacer pedagogía debe influir en el estudiante a través de la generación de nuevas actitudes, valores, juicios, sentimientos, capacidades, aptitudes, etc. Luego, desde la PC, queda claro que enseñar es transformar $y$, en consecuencia, emancipar (RodríguezRojo, 1997).

En plena relación con el valor 
emancipatorio de la educación, encontramos otro referente en Paulo Freire. Este es considerado uno de los padres de la pedagogía crítica. Para él la educación crítica aspira a cambiar la forma de ver el mundo, de educar $y$, en definitiva, de interactuar. Freire entiende que el papel fundamental de la educación es la liberación de los seres humanos y de los grupos sociales sometidos a relaciones de control, injusticia y explotación. Pretende crear individuos críticos y conscientes, que sean capaces de cambiar su realidad al adquirir un mayor grado de libertad, justicia e independencia. Este autor ha sido considerado la superación de la pedagogía de Piaget, Vygotsky y Kohlberg (Santos, 2008) con unas concepciones sobre la sociedad y la educación que, hoy más que nunca, siguen vigentes y en plenitud. Su gran obra, Pedagogía del oprimido (1970), recoge las bases sobre las que asentar el cambio hacia una conciencia crítica y transformadora. Acercándonos directamente a su legado, Freire nos da cuatro ideas básicas:

1. Educar es conocer críticamente la realidad.

2. Educar es comprometerse con la utopía de transformar la realidad.

3. Educar es formar sujetos de dicho cambio.

4. Educar es diálogo.

Algunas de las claves para entender la pedagogía que pretende transmitir Freire son, a partir de sus distintas obras, las siguientes:

- Superación de la educación bancaria, vigente todavía hoy en día, en la que hay una relación vertical caracterizada por la existencia de un sujeto que "da" los conocimientos, la educación, y otro que los recibe. Estos conocimientos son fijos y "ajenos" a los individuos. Freire defiende una relación horizontal en la que, 
mediante el diálogo entre educador y educando, se propicia la reflexión crítica a partir de conocimientos sociales. Nadie es el oprimido ni el opresor.

- Partida del cambio a partir de una situación límite, se precisa de una necesidad de superación. La educación como una práctica de la libertad.

- Superación de la estructura etnocéntrica. Lo social y lo individual no son aspectos excluyentes, son como un todo. Tal y como apuntan Buber (1998) y Marcel (2003), lo que nos hacer ser un yo singular es la presencia del tú. El diálogo debe ser siempre la base de todo cambio. Un diálogo crítico con la sociedad y con la comunidad en la que viven que propicia el crecimiento personal a partir de la relación con los demás.

- La pedagogía del oprimido
RIDAS

implica un posicionamiento político. Nunca será un aspecto neutro. Freire defiende la posición del político y del educador como figuras que deben luchar por una mejora común.

- Es vital la "concientización" que propiciará la transformación de la sociedad siempre a partir del pensamiento crítico.

En definitiva, lo que pretende la PC es una sociedad diversa que interactúa con todos sus miembros y a la que se ha llegado por medio de una educación que no margina a nadie. Se pretende crear personas críticas, que tomen parte en el desarrollo de su sociedad en busca de un ideal de superación.

En relación con este enfoque pedagógico se sitúan otros autores como Martí (2001), Jaspers (1958), Buber (1998), Marcel (2003) y Dussel (2002). Cada uno de ellos aporta su grano de arena a la defensa de la creación de una estructura social 


\section{RDISF}

crítica. La filosofía de Martí se encuentra estrechamente ligada a la PC al apoyar, entre otros ideales, la emancipación como punto de partida y el compromiso con la acción (Alvarado, 2007). Asimismo, el filósofo alemán Jaspers se posiciona en la lucha, el sufrimiento, como base de la existencia, las situaciones límite como punto de partida para "existir" (Santos, 2008). Buber y Marcel, por su parte, defienden la interacción como punto clave para el desarrollo existencial y Jaspers considera, a su vez, el límite existente en la comunicación existencial, base de esta concepción en la que se produce la creación y cambio del individuo a través de la comunicación mutua. A su vez, Dussel (2002), defiende que en esta pedagogía se propone el surgimiento de una conciencia éticocrítica $y$, además, que la producción de esta conciencia supone su propia liberación.

La PC bebe de todas estas aportaciones para, en el terreno de la praxis educativa, apuntar una serie de medidas de aplicación en los procesos concretos de enseñanzaaprendizaje (E-A). Como indica Rodríguez-Rojo (1997), la praxis concreta de la PC se preocupa ante todo de la formación de estructuras mentales, de la organización de los conocimientos fragmentados y de la crítica constructiva de los problemas cotidianos.

Para ello, en cada proceso de E-A deben plantearse actividades relacionadas con el análisis de prejuicios, sentimientos, saberes implícitos, experiencias y prácticas que el alumnado alberga en su esquema cognitivo previo. Luego, será necesario que los docentes provean a los discentes de procesos de debate, discusión y diálogo en torno a situaciones reales que conecten con su contexto más próximo. De esta manera, a través del contraste de pareceres y de la revisión y reflexión de conceptos, se brinda al alumnado un salto cualitativo en relación a las capacidades y facultades desarrolladas, ampliando y enriqueciendo sus posibilidades de 
comprensión de la realidad.

Así pues, superando los enfoques tradicionales, que limitan la enseñanza reglada al aula como espacio idóneo para transmitir conocimientos, la PC apuesta por el contexto para enfatizar la influencia de las subculturas, de las historias personales y de las diferencias particulares. De manera que se establece una relación dialéctica entre la escuela y la vida. La escuela sale al encuentro de los problemas vitales y la vida se aprovecha de las posibilidades de la escuela como espacio facilitador de la sistematización y reflexión de los conocimientos (Rodríguez-Rojo 1997).

\section{Aprendizaje servicio}

Las definiciones del ApS abundan en la literatura (Alliance Canadienne pour l'aprentissage par le service communautaire, 2010; CLAYSS, 2010; Community College National Center for Community Engagement, 2010; Furco y Billig, 2002; Halsted,
1998; Martínez, 2008; National and Community Service Trust Act, 1993; National Service-Learning Clearinghouse, 2010; Puig, Batlle, Bosch y Palos, 2007; Stanton, 1990). Analizándolas y revisando estas propuestas de definición, podemos apreciar que cada entidad o autor hace especial incidencia en un aspecto del ApS. Además es evidente que cada autor o entidad expresa la definición con su terminología propia, destacando matices diferentes en función de las características de su entorno. Con todo, existen términos clave que aparecen en casi todas ellas y que son los que verdaderamente definen el ApS.

Los términos clave que más aparecen en las definiciones son pedagogía, metodología, filosofía, estudiantes, profesores y comunidad, objetivos de aprendizaje académico y de desarrollo comunitario, responsabilidad cívica e implicación con la comunidad. En efecto, hay una coincidencia en todas las definiciones en expresar la idea de asociar o conjugar los contenidos curriculares 
con el servicio prestado a la comunidad.

Partiendo de estas premisas, concebimos el ApS como una metodología que procura el aprendizaje de contenidos académicos a la vez que se presta un servicio a la comunidad. Se enmarca dentro del aprendizaje experiencial, ya que su aplicación lleva aparejada la realización de prácticas reales. Procura dar significatividad a los aprendizajes, bien sean académicos o en el ámbito de la formación como ciudadanos, ya que tiene este doble objetivo.

Su aplicación se articula mediante el diseño de proyectos que deben estar perfectamente planificados para que se cumplan estos objetivos. Rompe con la tradición de la enseñanza en el aula: se debe salir a la sociedad y crear redes de colaboración con entidades diversas para encontrar la esencia de este tipo de forma de aprender. Sitúa al alumnado en el centro del proceso de E-A, Io convierte en el protagonista principal, dejando para el profesor el rol de guía, orientador y facilitador del proceso. Hace que aparezcan nuevos actores en un escenario tradicionalmente limitado a dos, profesor y alumno, al dar cabida a los profesionales de las entidades que juegan un papel decisivo en la formación del alumnado. Huye de propiciar aprendizajes memorísticos; muy al contrario, plantea tareas que van a obligar al alumnado a movilizar capacidades que difícilmente utilizarían en otros ámbitos.

Los rasgos del ApS pueden resumirse de acuerdo con tres dimensiones que a nuestro juicio constituyen su naturaleza: papel de los agentes implicados, características pedagógicas y papel de la sociedad.

En relación al papel de los agentes implicados:

- Alumnado y el profesorado planifican

- Reciprocidad y colaboración en el aprendizaje 
- Institución educativa comprometida con la formación y con la sociedad

- Profesorado "guía"

- Rol del colaborador (entidad social, profesionales) basado en su compromiso e implicación

En segundo lugar, en relación a las características pedagógicas:

- Receptores del servicio: colectivos en desventaja social o riesgo de exclusión

- Educación en conocimientos específicos y valores

- Utilidad social atendiendo una necesidad

- Pedagogía activa y reflexiva

- Alumnado activo en todos los momentos del proceso: planificación, ejecución y evaluación
- Formación deontológica, ciudadana, humana, personal y social

- Procesos de investigaciónacción

- Construcción de conocimientos en contextos de necesidad social

- Reflexión conjunta sobre la práctica

- Retroalimentación multidireccional (profesorado, receptores, agentes sociales...)

- Elaboración cooperativa y colaborativa del conocimiento

- Saber, saber hacer, saber ser y convivir

- Análisis crítico y social del entorno

En último lugar, en relación al papel de la sociedad: 
- Su prioridad es prestar un servicio a la comunidad mientras se aprende

- Receptores reconocen la necesidad de la prestación del servicio

- Mejora y transforma la sociedad a través del compromiso escuela/sociedad en busca de la justicia social

- Su objetivo es preparar al estudiante para abordar situaciones sociales y éticas en su futuro

Algunos de los elementos que destacan en la definición del ApS son el pensamiento crítico, la autoreflexión y la responsabilidad cívica, relacionados todos ellos estrechamente con las bases de la PC.

La práctica que se realiza mediante una intervención de ApS está directamente ligada con la comunidad en la que se lleva a cabo. Se busca la relación directa con el entorno con la finalidad de mejorarlo, de cambiarlo, al promocionar la participación en él. El cambio, siempre sobrevenido por la interacción, promueve un desarrollo del entendimiento del contexto $y$, a la vez, un cambio en la mentalidad.

En todas las intervenciones de ApS es imprescindible el contacto y el diálogo con la sociedad, siguiendo los preceptos de la PC, entre los que destacan la superación de la relación horizontal de la educación así como de la estructura etnocéntrica. Somos a partir de lo que hacemos, así como de con quién y cómo se lleva a cabo.

\section{Conexión entre aprendizaje servicio y pedagogía crítica}

Continuando con la idea inicial de este trabajo y habiendo sentado hasta aquí los preceptos básicos tanto de la PC como del ApS, el presente apartado avanza en el esclarecimiento de su relación. En la línea de alcanzar el objetivo emancipador y transformador de la PC, el ApS plantea una praxis educativa 
participativa, que a partir del diálogo escuela-sociedad favorezca el intercambio de pareceres a través del debate, la reflexión y la discusión en grupos, promoviendo diálogos en los que docentes y discentes se relacionan horizontalmente.

Entre los elementos más destacados sobre los que se establece la conexión de la PC con el ApS, siguiendo los postulados de la PC que plantea Freire, podemos destacar:

- Relación horizontal de la educación. Mediante los programas de ApS todos aprenden de todos, es decir, la interacción ofrece una fuente de conocimiento a los todos los participantes. La diversidad se considera en este sentido un beneficio. El conocimiento y el entendimiento que se produce al establecer relaciones con colectivos desfavorecidos provoca un cambio de identidad que, posteriormente, se convertirá en un cambio de actitud.
- Situación límite como punto de partida del cambio. La desigualdad y la marginalidad que existe en nuestra sociedad marca el punto de partida hacia una comunidad mejor. El trabajo con asociaciones e instituciones sociales previene este olvido y promueve la participación de la sociedad con estos colectivos.

- Diálogo como base del cambio. Lo social y lo individual como un todo. La interacción, una vez más, juega el papel más importante del ApS como práctica educativa que nos llevará a cambiar la sociedad.

- Implicación de un posicionamiento político. No existe una neutralidad en las experiencias de ApS. Al formar parte de la comunidad se produce una toma de conciencia $y$, por consiguiente, un posicionamiento social $y$ político. 
- Existencia de un compromiso de transformación. El cambio nunca se puede producir si no existe un compromiso. En el $A p S$, éste se adquiere mediante la interacción con colectivos en situación desfavorecida. Este compromiso promueve el cambio y la transformación hacia una sociedad mejor.

- La educación forma a los sujetos del cambio. Los individuos que llevan a cabo experiencias de ApS se sumergen en las comunidades. Deben conocerlas, igual que la sociedad en la que viven, para que aparezcan los efectos deseados del uso pedagógico del ApS y no caer en situaciones anómalas que podrían conllevar situaciones contrarias a lo esperado. Este es el camino para formar personas críticas y consecuentes con su contexto social, aspectos que van a suponer el motor del cambio.
Basándonos en esta fundamentación, presentamos a continuación un cuadro con los principales nexos de unión entre la metodología del ApS y el paradigma de la PC.

Cuadro 1. Similitudes entre pedagogía crítica y aprendizaje servicio

\begin{tabular}{|c|c|}
\hline PEDAGOGÍA CRÍTICA & APRENDIZAJE SERVICIO \\
\hline $\begin{array}{l}\text { Relación horizontal } \\
\text { de la educación: } \\
\text { todos aprendemos de } \\
\text { todos }\end{array}$ & $\begin{array}{l}\text { Profesorado "guía" } \\
\text { Alumnado activo en todos los } \\
\text { momentos del proceso: } \\
\text { planificación, ejecución y } \\
\text { evaluación }\end{array}$ \\
\hline $\begin{array}{l}\text { Situación límite como } \\
\text { punto de partida del } \\
\text { c a m b i o : r I a } \\
\text { desigualdad marca el } \\
\text { punto de partida del } \\
\text { cambio }\end{array}$ & $\begin{array}{l}\text { Receptores del servicio: } \\
\text { colectivos en desventaja } \\
\text { social o riesgo de exclusión } \\
\text { Construcción } \\
\text { conocimientos en contextos } \\
\text { de necesidad social } \\
\text { Receptores reconocen la } \\
\text { necesidad de la prestación } \\
\text { del servicio }\end{array}$ \\
\hline $\begin{array}{l}\text { Diálogo como base } \\
\text { del cambio y de la } \\
\text { educación. Lo social y } \\
\text { lo individual como un } \\
\text { todo: relación e } \\
\text { interacción con la } \\
\text { comunidad como }\end{array}$ & $\begin{array}{l}\text { - Reciprocidad e n e I } \\
\text { aprendizaje y colaboración } \\
\text { Institución educativa } \\
\text { comprometida con formación }\end{array}$ \\
\hline
\end{tabular}




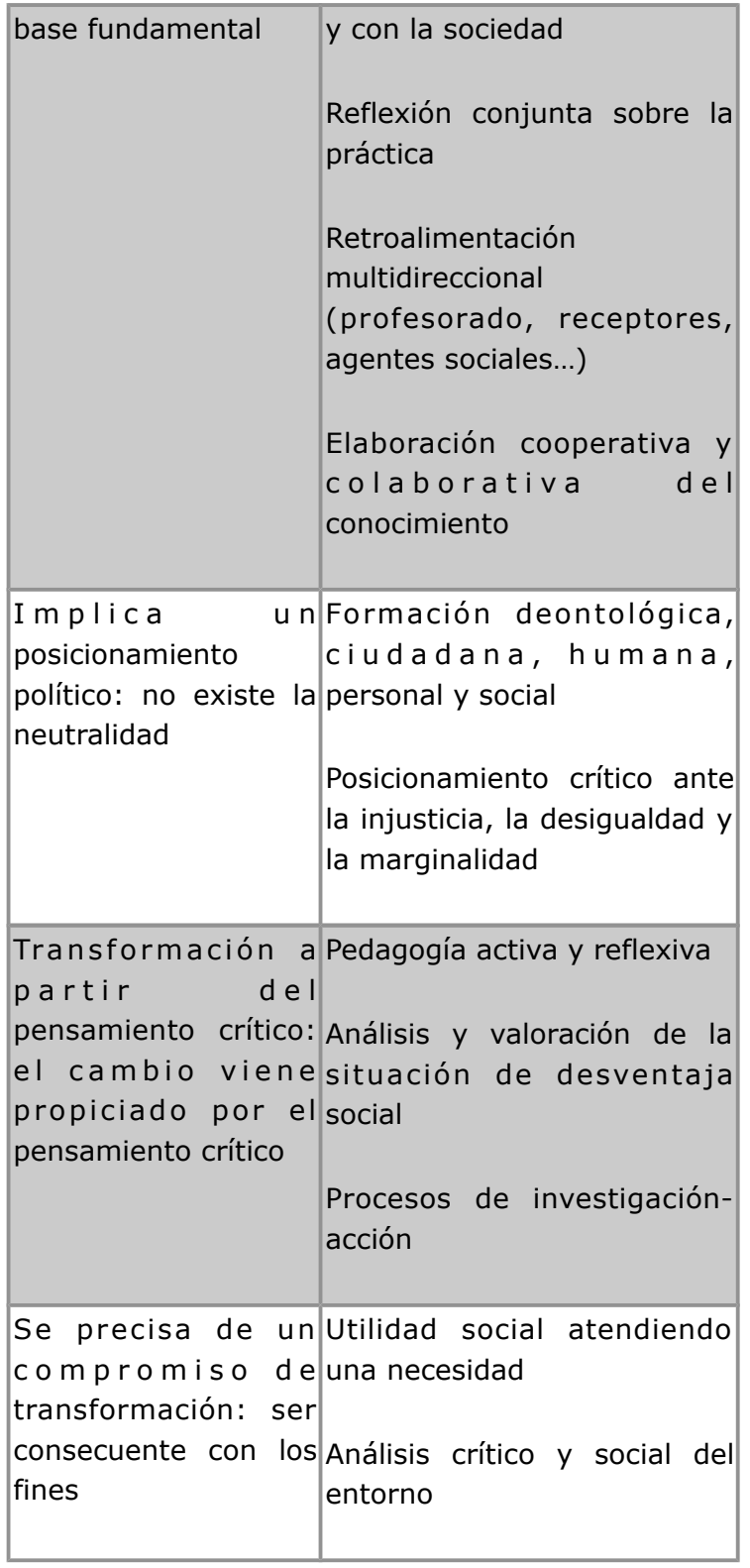

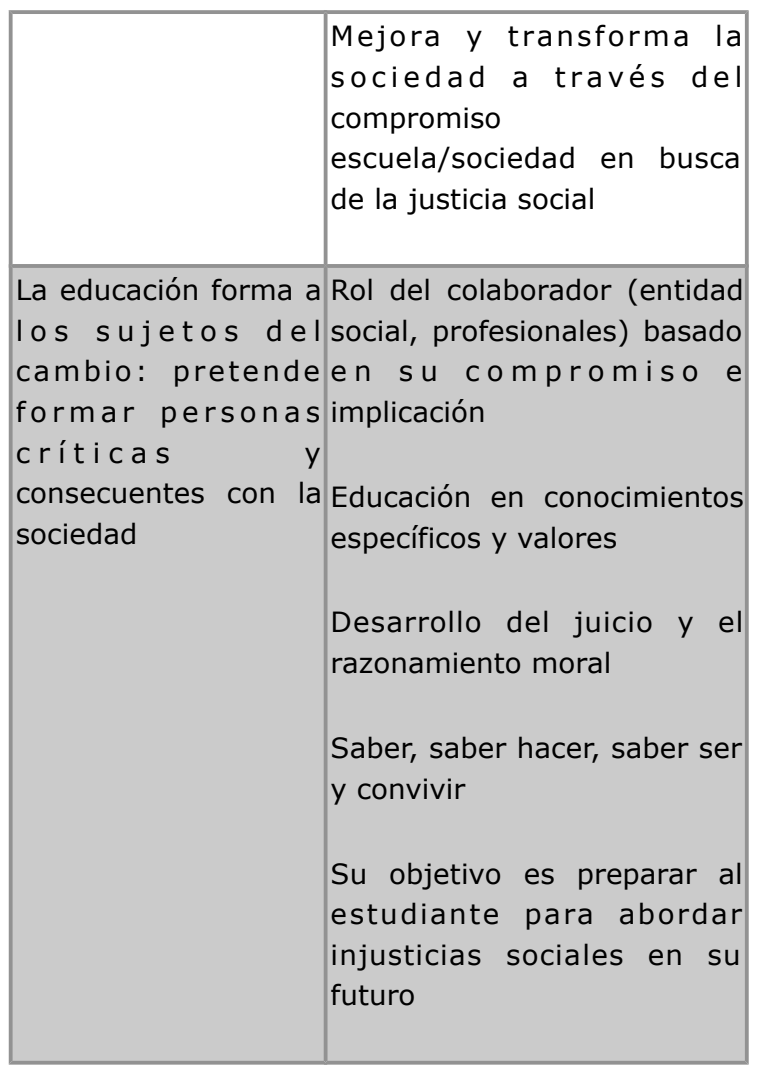

Fuente: Elaboración propia

Tras este resumen, observamos la alta coincidencia entre la PC y el ApS, lo que nos muestra el potencial de la metodología del ApS para formar personas críticas y conseguir una transformación de la sociedad, ya que cada uno de los aspectos referidos de la $\mathrm{PC}$ se ve reflejado en el ApS. 
Trasladando esta fundamentación al ámbito de la experiencia real, exponemos a continuación una revisión de resultados de investigación obtenidos en diferentes experiencias de ApS, en las que se refleja su relación con los rasgos básicos de la PC. Finalmente, para mayor concreción, contrastaremos esta relación vinculando los atributos expuestos en el cuadro 2 con los resultados tangibles de una investigación concreta, centrada en los efectos del ApS sobre el desarrollo de la ciudadanía.

\section{1.-Estudios sobre los efectos del aprendizaje servicio}

La aplicación del ApS se ha llevado a cabo a nivel mundial, aunque no sin problemas o retos a los que han tenido que hacer frente. Las experiencias de las que se tiene constancia se centran, sobre todo, en la educación superior. Los estudios en los que se analizan los efectos que la metodología ha tenido sobre el alumnado muestran efectividad en dimensiones como los aprendizajes técnicos o formales, el sentimiento de autoeficacia, el entendimiento cultural, la adquisición y práctica de valores y el desarrollo del pensamiento crítico (Bernadowski, Perry y Del Greco, 2013; Billig, 2002; Carrington e Iyer, 2011; Carrington y Saggers, 2008; Chambers y Lavery, 2012; Chang, Anagnostopoulos y Omae, 2011; Eyler, Giles, Stenson y Gray, 2001; Gil, 2012; Miller y González, 2010; Yang, 2012; Youniss, Yates, 1997; Zhang, Zeller, Griffith, Metcalf, Williams, Shea y Misulis, 2011). Unas amplias revisiones de estos efectos son las realizadas por Conway, Amel y Gerwien (2009) y Yorio y Ye (2012).

Desde el interés del presente artículo cabe destacar la existencia de estudios que han medido los efectos de la aplicación del ApS sobre la ciudadanía. Diversas revisiones han aportado evidencias de mejora en la ciudadanía a nivel global (Billig, 2002; Perry y Katula, 2001; Youniss y Yates, 1997). Algunos investigadores han visto efectos concretados en un mejor ejercicio de la ciudadanía y de la 
responsabilidad ciudadana (Ammon, Furco, Chi y Middaugh, 2002; Astin y Sax, 1998; Covitt, 2002; Kahne y Westheimer, 2002; Levine y Lopez, 2002; Moely, McFarland, Miron, Mercer y Ilustre, 2002). Otros autores han proporcionado muestras de cambios positivos en actitudes cívicas y conductas prosociales (Billig, 2002; Billig, Root y Jesse, 2005; Kim y Billig, 2003; Melchior, 1999; Morgan y Streb, 1999; Scales, Blyth, Berkas y Kielsmeier, 2000).

Comovemos, los efectos documentados derivados de la aplicación del ApS indican claramente que el alumnado adquiere aprendizajes que van más allá de la vertiente técnica, adentrándose en aspectos que son comunes con los objetivos de la PC. Desarrolla el entendimiento cultural y el pensamiento crítico, permitiendo la movilización de valores y propiciando el ejercicio de la ciudadanía en el sentido amplio del término (responsabilidad social, participación y autonomía). Sin duda, estos son efectos compartidos por la PC, pudiéndose afirmar que el ApS es una forma de esta.

\subsection{Un ejemplo práctico}

Para abundar en lo anterior, describimos de un modo más detallado una investigación sobre los efectos del ApS sobre la movilización de valores relacionados con la ciudadanía. Concretamente se expone una experiencia de ApS diseñada y aplicada en el Área de Didáctica de la Expresión Corporal de la Universitat Jaume I. Se trata de una aplicación basada en el documento "Estándares de Aprendizaje-Servicio K-12 para la Práctica de Calidad" propuesto en 2008 por el National Youth Leadership Council de Estados Unidos. En esta aplicación didáctica han intervenido, por una parte, el alumnado de la asignatura Bases anatómicas y fisiológicas del movimiento, perteneciente a la titulación de maestro de la Universitat Jaume I, y por otra parte, varias entidades sociales centradas en la atención a personas con dificultades motrices derivadas de diversas 
patologías anatómicas y / o fisiológicas. A través de esta metodología el alumnado universitario ha aplicado los conocimientos específicos de la asignatura en cuestión, para prestar un servicio a la comunidad. A través de la misma, el alumnado universitario aprendió los conocimientos de la asignatura y desarrolló valores y actitudes cívicas (Gil, 2012).

Se implementó durante 11 semanas con una implicación de 12,3 horas semanales por parte del alumnado (54 participantes). Se crearon alianzas sociales con cuatro entidades dedicadas a la atención de personas con dificultades motrices, pretendiendo a umentar la comprensión de la diversidad que presenta el mundo de la discapacidad. El alumnado fue el protagonista de la aplicación didáctica al fomentar su autonomía en la toma de decisiones en todas las fases del proceso de E-A. En el programa se establecieron varios sistemas de supervisión y seguimiento: reuniones periódicas grupales $\mathrm{y} / \mathrm{o}$ del grupo- clase con diálogos y debates, seguimiento diario con documentos remitidos electrónicamente, y visitas periódicas a las entidades donde se prestaba el servicio. Asimismo se articularon sistemas de reflexión para que el alumnado tomara consciencia de los cambios acaecidos en su estructura cognitiva inicial.

Paralelamente a esta experiencia se realizó una investigación cualitativa (los datos se recogieron a través de la cumplimentación de diarios personales, grupos de debate posteriores a las sesiones prácticas con los receptores del servicio y tutorías individuales y grupales con el profesorado), de la que se obtuvieron resultados sobre cómo había cambiado la estructura de valores y de personalidad del alumnado que participó. Los cambios se manifestaron en los siguientes ámbitos:

Conformidad con lo socialmente correcto. Se potenció el respeto hacia las personas con autoridad en el ámbito académico, la aceptación de 
las normas de convivencia en casa y el respeto hacia los asuntos de los compañeros. El pensamiento crítico en este punto se manifestó cuando el alumnado fue capaz de acatar las normas sociales razonables $y$ diferenciarlas de aquellas $\sin$ base lógica, que eran cuestionadas de forma fundamentada.

Sensibilidad social. La empatía hacia otros, la capacidad de escuchar, comprender y descubrir cualidades en los demás, la satisfacción por compartir la alegría cuando a otra persona algo le sale bien y por estar con los compañeros fueron los aprendizajes logrados en este campo. Los estímulos vividos despertaron la conciencia social del alumnado, a partir de la observación del modelo de actuación de los profesionales de las entidades respecto a su implicación y compromiso. Estos resultados son compatibles con el planteamiento de Ia PC respecto al compromiso consustancial con la necesidad del cambio social. La apertura de los centros educativos a la sociedad y la cantidad y riqueza de las interacciones personales que el caso de la experiencia supuso, condujo a la potenciación de valores como la empatía, el diálogo, la capacidad de esfuerzo, la superación, valorar a los demás, etc., todo ello relacionado con el cambio de la estructura de valores inicial del alumnado.

Ayuda y colaboración. Se incrementó la generosidad con los demás, la capacidad de trabajar en grupo y de ayudar a los componentes de un equipo y de dedicar tiempo a los demás si lo necesitan. Se asumieron valores como el consenso, la integración, la comunicación, el estímulo al trabajo, etc., desde la perspectiva de la relación horizontal de la estructura del proceso de E-A en el ApS, cuestión absolutamente ligada a los principios básicos de la PC.

Seguridad y firmeza en la interacción. Aprendieron a reconocer errores, defender derechos y dialogar con las personas con las que se tiene algún problema cara a cara para solucionarlo. Experimentaron un aumento de seguridad en ellos 
mismos, manifestada en una actitud más beligerante ante el sentimiento de injusticia social. Estos resultados se alinean directamente con el planteamiento dialógico que defiende la PC, así como con la adopción de un posicionamiento no neutral ante los conflictos, siempre desde una perspectiva de análisis crítico de la realidad vivida.

Responsabilidad social. El alumnado mostró implicación personal para actuar ante problemas sociales, aceptándolos como parte de uno y aceptando la responsabilidad individual. Despertó la capacidad de asumir obligaciones sin que nadie se lo tuviera que recordar y de implicarse en proyectos para mejorar la sociedad. Relación entre la responsabilidad individual y la social.

De estos resultados se desprende una alineación clara de los postulados de la PC con el impacto que provoca la aplicación del ApS sobre el estudiantado. Sin duda, es palpable cómo la relación e interacción con la comunidad constituye la base fundamental para la transformación de la misma. En definitiva, en este artículo nos habíamos marcado el objetivo de esclarecer la conexión entre la PC y la metodología del ApS. Tras la argumentación expuesta en los primeros apartados y la constatación de los mismos a través del análisis de resultados de investigaciones, podemos afirmar finalmente que el ApS es una metodología que encaja dentro del paradigma de la PC.

\section{Referencias bibliográficas}

Alvarado, M. (2007). José Martí y Paulo Freire: aproximaciones para una lectura de la pedagogía crítica. Revista Electrónica de Investigación Educativa. 9(1). Recuperado de: http://redie.uabc.mx/index.php/redie Larticle/view/157

Ammon, M., Furco, A., Chi, B. y Middaugh, E. (2002). Service-learning in California: A profile of the CalServe service-learning partnerships (19972000 ). National Service-Learning Claringhouse Resources. Recuperado 
de:

http://servicelearning.org/filemanage r/

Astin, A; Sax, L. (1998). How undergraduates are affected by service participation. Journal of College Student Development. 39(3), 251-263.

Bernadowski, C., Perry, R. y Greco, R. Del. (2013). Improving Preservice Teachers' Self-Efficacy through Service Learning: Lessons Learned. International Journal of Instruction. 6 No.2(2).

Billig, S. (2002). Support for K-12 Service-Learning Practice: A Brief Review of the Research. Educational Horizons. 80(4), 184-190.

Billig, S., Root, S; Jesse, D. (2005). The impact of participation in servicelearning on high school students' civic engagement. College Park, MD: Center for Information and Research on Civic Engagement and Learning

Buber, M. (1998). Yo y tú. Madrid:
Caparrós.

Canadian Alliance for Comunity Service-Learning. (2010). Recuperado de:

http://www.communityservicelearnin g.ca/en/

Carrington, S., y Iyer, R. (2011). Service-Learning Within Higher Education: Rhizomatic Interconnections Between University And The Real World. Australian Journal of Teacher Education. 36(6).

Carrington, S., Saggers, B. (2008). Service-learning informing the development of an inclusive ethical framework for beginning teachers. Teaching and Teacher Education. 24(3), 795-806.

Centro Latinoamericano de Aprendizaje y Servicio Solidario(2010). Recuperado de: http://www.clayss.org.ar

Chambers, D .,Lavery, S. (2012). Service-Learning : A Valuable Component of Pre- Service Teacher 
Education. Australian Journal of Teacher Education. 37(4).

Chang, S., Anagnostopoulos, D., Omae, H. (2011). The multidimensionality of multicultural service learning: The variable effects of social identity, context and pedagogy on pre-service teachers' learning. Teaching and Teacher Education. 27(7), 1078-1089.

Conway, J., Amel, E .,Gerwien, D. (2009). Teaching and Learning in the Social Context: A Meta-Analysis of Service Learning's Effects on Academic, Personal, Social, and Citizenship Outcomes. Teaching of Psychology. 36(4), 233-245.

Covitt, B. (2002). Motivating environmentally responsible behavior through servicelearning. En S.H. Billig y A. Furco (Eds.), Advances in service-learning research: Servicelearning through a multidisciplinary lens (pp. 177-197). Los Angeles, CA: RMC Research Corp.

Dussel, E. (2002). Ética de la liberación en la edad de la globalización y de la exclusión. Madrid: Trotta.

Eyler, J., Giles, D., Stenson, C. M., Gray, C. (2001). At A Glance : What We know about The Effects of Service-Learning on College Students, Faculty, Institutions and Communities, 1993- 2000 : Third Edition. Corporation for National Service Learn and Serve America National Service Learning Clearinghouse, 1993-2000.

Furco, A.; Billig, S.H. (2002). Service Learning. The essence of the Pedagogy. Greenwic. C.T. Ley sobre Servicio Nacional y Comunitario: "National and Community Service Trust Act". (1990). Ratificada y ampliada en 1993.

Gil, J. (2012). El aprendizaje-servicio en la enseñanza superior: una aplicación en el ámbito de la educación física. Tesis doctoral. Universitat Jaume I de Castellón.

Halsted, A. (1998). Educación 
redefinida: la promesa del Aprendizaje-Servicio. En: Ministerio de Cultura y Educación. Dirección de Investigación y Desarrollo Educativo. El servicio a la comunidad como aprendizaje escolar. Actas del 1er Seminario Internacional "Educación y Servicio Comunitario", Buenos Aires, 1998, pp. 23-24.

Kahne, J.; Westheimer, L. (2002, mayo). The limits of efficacy: Educating citizens for democratic action. Comunicación presentada en el Annual Meeting of the American Political Science Association. Boston, MA.

Kim, W.; Billig, S.H. (2003) Colorado Learn and Serve Evaluation. Research Report. Denver, CO: RMC Research Corporation.

Levine, P.; López, M.H. (2002). Youth voter turnout has declined, by any measure (CIRCLE). College Park, MD: The Center for Information \& Research on Civic Learning \& Engagement.
Martínez, M. (2008). Aprendizaje servicio y responsabilidad social de las universidades. Barcelona: ICEOctaedro.

Melchior, A. (1999). National Evaluation of Learn and Serve America. Waltham, MA: Brandeis University.

Miller, K; Gonzalez, A. (2010). Domestic and international service learning experiences : A comparative study of pre-service teacher outcomes. Issues in Educational Research. 20(1), 29-38.

Moely, B., McFarland, M., Miron, D., Mercer, S.; Ilustre, V. (2002). Changes in college students' attitudes and intentions for civic involvement as a function of servicelearning experiences. Michigan Journal of Community Service Learning. 9, 1826

Morgan, W.; Streb, M. (1999). How quality service-learning develops civic values. Bloomington, IN: Center for Participation and Citizenship. 
Habermas, J. (1982). Conocimiento e interés. Madrid: Taurus.

Habermas, J. (1989). El discurso filosófico de la modernidad. Madrid: Editorial Taurus.

Habermas, J. (1991). Conciencia moral y acción comunicativa. Barcelona: Península.

Habermas, J. (2002). El futuro de la naturaleza humana. ¿Hacia una eugenesia liberal? Barcelona: Paidós.

Jaspers, K. (1958). Filosofía. Madrid: Revista de Occidente-Ediciones de la Universidad de Puerto Rico.

López-Pastor, V. M. (2002). (Coord). Algunas reflexiones sobre educación física y pedagogía crítica. Retos. Nuevas tendencias en Educación Física, Deporte y Recreación. 2, pp. 30-35.

Marcel, G. (2003). Ser y tener. Madrid: Caparrós.

Perry, J.; Katula, M. (2001). Does service affect citizenship?
Administration \& Society. 33, 330365.

Puig, J. M., Batlle, R., Bosch, C. ; Palos, J. (2007). Aprendizaje servicio. Educar para la ciudadanía. Barcelona: Ministerio de Educación y Ciencia, Octaedro.

Rodríguez-Rojo, M. (1997). Hacia una didáctica crítica. Madrid: La Muralla.

Santos, M. (2008). Ideas filosóficas que fundamentan la pedagogía de Paulo Freire. Revista Iberoamericana de Educación. 46,155-173.

Scales, P. C., Blyth, D., Berkas, T.; Kielsmeier, J. (2000). The Effects of Service Learning on Middle School Students' Social Responsibility and Academia Success. The Journal of Early Adolescence. 20(3), 332-358.

Stanton, T. K. (1990). Service Learning: Groping toward a definition. En: KENDALL, J. C. and Associates, Combining Service and Learning: a resource book for community and public service, 65-67. Vol 1. Raleigh 
North Carolina: National Society for Internships and Experiential Education.

Yang, Y.-T. C. (2012). Cultivating critical thinkers: Exploring transfer of learning from pre-service teacher training to classroom practice. Teaching and Teacher Education, 28(8), 1116-1130.

Youniss, J.; Yates, M. (1997). Community service and social responsibility in youth. Chicago: University of Chicago Press.

Yorio, P. L.; Ye, F. (2012). A Meta-
Analysis on the Effects of ServiceLearning on the Social, Personal, and Cognitive Outcomes of Learning. Academy of Management Learning \& Education. 11(1), 9-27.

Zhang, G., Zeller, N., Griffith, R., Metcalf, D., Williams, J., Shea, C.; Misulis, K. (2011). Using the Context, Input, Process, and Product Evaluation Model (CIPP) as a Comprehensive Framework to Guide the Planning, Implementation, and Assessment of Service-learning Programs.Journal of Higher Education Outreach and Engagement. 15(4), 57-84. 